\title{
EPIGÉNESIS DE LA RAZÓN PURA Y ESPACIALIZACIÓN DEL TIEMPO EN KANT
}

\author{
Camilo Estay Muñoz ${ }^{1}$ \\ Universidad de Santiago, Chile
}

\begin{abstract}
Resumen: En este artículo se intentará dilucidar la expresión 'epigénesis de la razón pura' de Kant en relación con el orden de fundamentación trascendental de constitución de la objetividad, tal y como es presentado en la Crítica de la razón pura. De manera especial, se examinará la relación de fundamentación que existe entre el tiempo y el espacio. Se defenderá la tesis según la cual el espacio es lógica e intuitivamente prioritario respecto del tiempo, para lo cual se acudirá al pasaje titulado 'Refutación al idealismo'. Junto con esto, se llevará a cabo una interpretación del Esquematismo y las Analogías de la Experiencia que dé cuenta de que, además, se puede inferir una espacialización del tiempo en el pensamiento de Kant.
\end{abstract}

Descriptores: Epigénesis de la razón pura $\cdot$ Espacio $\cdot$ Tiempo $\cdot$ Sentido externo $\cdot$ Sentido interno $\cdot$ Esquematismo.

Abstract: This article will attempt to elucidate Kant's expression 'epigenesis of pure reason' in relation to the order of the transcendental foundation of constitution of objectivity, as presented in the Critique of Pure Reason. By using the passage entitled 'Refutation of idealism', the fundamental relationship existing between time and space will be examined, and the thesis according to which space has logically and intuitively priority over time will be defended. Along with this, the article proposes an interpretation of the Schematism and Analogies of Experience, which is able to give an account of a spatialization of time in Kant's thought.

Keywords: Epigenesis of Pure Reason $\cdot$ Space $\cdot$ Time $\cdot$ Outer Sense $\cdot$ Inner Sense $\cdot$ Schematism.

Enviado: 22/09/2020. Aceptado: 27/11/2020

\section{INTRODUCCIÓN}

En la introducción a la Crítica de la razón pura ${ }^{2}$ Kant sostiene que "aunque todo nuestro conocimiento comience con la experiencia, no por eso surge todo él de la experiencia" (KrV, B1). Inmediatamente después explica que el conocimiento

\footnotetext{
${ }^{1}$ Licenciado en Filosofía, Pontificia Universidad Católica de Chile. E-mail: csestay@uc.cl

${ }^{2}$ En este trabajo se citará la Crítica de la razón pura como es habitual: con las siglas 'KrV' seguidas de la correspondiente edición (A y/o B) y número de página. El resto de las obras de Kant serán citadas según la notación canónica de la Akademie der Wissenschaften zu Berlin. Por otra parte, las traducciones de los textos en inglés serán propias. En cuanto a la traducción de la $\mathrm{KrV}$, se utilizará la versión de Pedro Ribas, aunque se tendrá en consideración igualmente la versión de Mario Caimi.
} 
cuyas fuentes se encuentran en la experiencia es a posteriori, mientras que aquel que no proviene de la misma es a priori. Podría en esta parte identificarse, según la clásica distinción cartesiana de las ideas, a priori con innato. En este sentido, podría pensarse que las formas puras de la sensibilidad y el entendimiento se hallan preformadas en el sujeto y listas para ser aplicadas a la materia del conocimiento. No obstante, es evidente que este no es el pensamiento de Kant ${ }^{3}$. Así pues, en un texto posterior a la Crítica y conocido como Respuesta a Eberhard, Kant es explícito a la hora de hablar sobre el origen de las formas puras, tanto de la sensibilidad como del entendimiento; menciona, pues, que "la Crítica no admite en lo absoluto representaciones innatas o implantadas. Ya sea que pertenezcan a la intuición o a los conceptos del entendimiento, se consideran adquiridas. Pero hay también una adquisición originaria (...). ${ }^{\prime 4}$ (Ak, 8:221).

Es claro que las representaciones puras a priori no pueden ser adquiridas empíricamente, pues en tal caso la misma noción de a priori carecería de sentido. ¿A qué se refiere Kant, entonces, con ‘adquisición originaria’? Una primera explicación la encontramos en su etapa precrítica, específicamente en la Dissertatio, donde señala, refiriéndose a espacio y tiempo, que "son adquiridos, ciertamente no por abstracción de los objetos de los sentidos (...), sino por la misma acción de la mente, que coordina lo que es sentido según leyes permanentes" (Ak, 2:406). Según esto, entonces, hay un dinamismo interno de la mente que origina las formas puras.

Ahora bien, este dinamismo no es completamente autónomo, ya que opera con ocasión de la materia que es recibida. Es en este contexto donde hace sentido una observación introducida por Kant hacia el final de la Deducción Trascendental (B), y que consiste en señalar que las categorías hacen posible la experiencia en "un sistema, por así decirlo, de epigénesis de la razón pura" ${ }^{5 "}(\mathrm{KrV}, \mathrm{B} 167)$. En el párrafo

\footnotetext{
${ }^{3}$ Llama la atención que muchos comentaristas de Kant, entre ellos algunos de los más importantes, hayan tenido que aclarar el no-innatismo del filósofo en reiteradas ocasiones y sin dirigir su crítica a ningún autor contemporáneo que defienda lo contrario (Véase, por ejemplo, Kemp Smith:1923, p. 91 y ss., Paton:1936, p. 78 y ss., De Pierris:1987, pp. 285-305, Zoeller:1989, pp. 222-235, Waxman:1991, p. 44 y ss., y Franck:2007, p. 70 y ss.). Al parecer este empeño se debe a que existe una idea generalizada de que Kant es innatista, es decir, una idea que circunda el mundo académico, pero que no es defendida seriamente por casi ningún comentarista especializado en Kant; la idea de que Kant es innatista tiene sus antecedentes en algunos de los enemigos intelectuales contemporáneos de Kant, entre los cuales destaca la figura de Johann Eberhard. Para una exposición de la controversia Kant-Eberhard sobre el innatismo en la Crítica, véase Allison:1973 p. 82 y ss.

${ }^{4}$ Lo que Kant, en cambio, sí considera innata, es nuestra especial capacidad receptiva en la sensibilidad y la espontaneidad en el entendimiento; no así sus formas (Cf. ídem).

${ }^{5}$ Aquí 'razón pura' no es usado en el sentido más restringido, a saber, como la facultad de las ideas trascendentales que son estudiadas en la Dialéctica Trascendental, sino más bien en el amplio sentido, incluyendo todos los elementos trascendentales a priori de la mente (para una nota de los sentidos de 'razón pura' en la Crítica, Cf. Kemp Smith:1923, p.2). De este modo, se puede identificar 'razón pura' con el término Gemüt, el cual es usado por Kant para designar al conjunto de todas las facultades mentales: de conocimiento, de placer y displacer, y de desear (Cf. Ak 5:197 y s.).
} 
siguiente Kant contrasta este sistema con el que denomina de preformación de la razón pura, ${ }^{6}$ el cual, según el filósofo, conduce directamente a un subjetivismo que invalidaría la necesidad con la que se aplican los conceptos puros a los fenómenos.

Como se puede apreciar, Kant está usando un lenguaje que hereda de la biología de su tiempo para hablar de elementos metafísicos, un lenguaje que, en este caso, no debe ser tomado en un sentido meramente ilustrativo o alegórico, sino más bien arquitectónico en relación con el sistema que intenta explicar. De hecho, no es exagerado sostener que "la contribución de las ciencias de la vida es histórica o biográficamente integral a la emergencia del idealismo trascendental" (Olson, 2007, p. 795), dado el gran interés que le reviste a Kant el tema de la vida. ${ }^{7}$ De esta manera, descifrar a qué se refiere con 'epigénesis de la razón pura' podría ayudarnos a comprender con mayor profundidad cuál es el modelo representacional de la mente que nos ofrece en la Crítica.

Antes que todo debemos definir qué son preformacionismo y epigenetismo en la biología, pues es la única manera de estar en posición de interpretar correctamente las palabras de Kant, en circunstancias de que este mismo nunca explica completamente en ningún escrito cómo habría que entender la epigénesis en su teoría de la actividad mental. Así pues, podemos decir, grosso modo, que en la biología del desarrollo -especialmente en la embriogénesis- el preformacionismo es una teoría que entiende el desarrollo de los organismos vivos como un proceso de despliegue de una estructura preexistente. En este sentido, un preformacionista tradicional concebiría el desarrollo básicamente como crecimiento, vale decir, como un cambio meramente cuantitativo de dicha estructura. Por su parte, el epigenetismo considera que el desarrollo es un proceso complejo de ordenamiento de la materia, inicialmente amorfa, hacia una forma biológica estructurada y que se activa con ocasión del contacto con el ambiente (Cf. Vecchi y Hernández, 2015, p. 578). De este modo, el epigenetista entiende el desarrollo, no sólo como crecimiento, sino como un proceso conducido por ciertos principios a partir de los cuales emergen las formas o rasgos de los organismos. Un hecho interesante aquí es que la epigénesis implica la emergencia de, a lo menos, una propiedad -un rasgo-, pero esta emergencia tiene su razón de ser en órdenes materiales distintos: el orden físico produce la emergencia de un orden químico, y sólo entonces pueden emerger rasgos biológicos que suponen los niveles anteriores. A su vez, si de un ser más complejo se trata, hay rasgos biológicos que son condiciones necesarias para que emerjan otros, tal y como

\footnotetext{
${ }^{6}$ Aquí la referencia es principalmente a Crusius, tal y como se evidencia en Reflexionen 4275 (Ak, 17:492). Sin embargo, indirectamente Kant ataca también en este pasaje el innatismo de Leibniz.

${ }^{7}$ No sólo aparece este tema en su conocida Crítica del juicio (Ak, 5:423 y ss.), sino también a lo largo de sus lecciones de metafísica: Metaphysik K2 (Ak, 28:760 y ss.), Metaphysik K3 (Ak, 29:1031 y ss.), Metaphysik Dohna (Ak, 28:684 y ss.), etc.
} 
ocurre en la relación entre los rasgos genotípicos y fenotípicos. En consecuencia, la idea de jerarquía fundacional es inseparable de la noción de epigénesis.

Dicho lo anterior, y sin acudir a una naturalización de Kant al estilo de Lorenz, ${ }^{8}$ ¿qué clase de jerarquías se pueden apreciar en el orden de fundamentación trascendental kantiano? Este trabajo se limitará únicamente a esclarecer el orden jerárquico existente entre espacio y tiempo. De este modo, el plan de trabajo es el siguiente: (i) en una primera sección se defenderá la idea según la cual el espacio es lógica e intuitivamente anterior al tiempo; esto se logrará recurriendo a varios pasajes de la Crítica, pero haciendo especial énfasis en uno de los apartados agregados en la segunda edición y que se titula Refutación al idealismo. (ii) Una vez conseguido lo anterior, en la segunda sección se hará un ejercicio interpretativo del Esquematismo Trascendental y las Analogías de la Experiencia en el que se intentará mostrar que el espacio, no sólo es anterior al tiempo, sino que originariamente lo produce, o, dicho de otro modo, el tiempo es espacializado en el modelo epigenético de la razón pura kantiano.

\section{RELACIÓN DE PRIORIDAD ENTRE ESPACIO Y TIEMPO}

¿Qué son espacio y tiempo para Kant? Para definirlo de manera muy sintética, son intuiciones puras que ordenan distintos dominios de manifestación de los fenómenos en la sensibilidad. En el caso del espacio, este es la forma del sentido externo, mientras que el tiempo lo es del sentido interno (Cf. KrV, A22-23/B37). De este modo, se puede decir que en el espacio se representan estados de cosas, mientras que en el tiempo se intuyen los propios estados mentales.

Es habitual la opinión según la cual, en la Crítica, el tiempo aparece como una intuición pura prioritaria con respecto al espacio. Esta interpretación tiende a ser persuasiva en tanto que se evidencia una preocupación especial de Kant por el tema del tiempo. Así pues, no sólo aparece al comienzo del texto, i. e., en la Estética Trascendental, sino que también vemos un profundo e interesante tratamiento de la temporalidad en la Deducción Trascendental (A) ${ }^{9}$ y en la sección del Esquematismo Trascendental. A su vez, en los Principios del Entendimiento Puro se puede observar que Kant dedica la mayor parte del texto al tratamiento de sus Analogías de la Experiencia, donde aparece su concepción más elaborada sobre el tiempo, pues incluye todos los análisis anteriores en orden a sistematizarlos y ofrecernos una distinción entre el tiempo subjetivo y el tiempo objetivo. Ahora bien,

\footnotetext{
${ }^{8}$ Véase especialmente Lorenz, K. "La teoría kantiana de lo apriorístico desde el punto de vista de la biología actual" (1941).

${ }^{9}$ Todo el argumento de la primera vía de la Deducción Trascendental (A) -entiéndase la 'doctrina de la triple síntesis'- descansa sobre el orden temporal en el que se nos presenta la variedad en la intuición.
} 
más allá de este motivo que por sí mismo no es concluyente, la razón principal que se ha esgrimido para sostener la prioridad del tiempo es que el mismo Kant sostiene que "el tiempo es la condición formal a priori de todos los fenómenos. El espacio, en cuanto forma pura de toda intuición externa, se refiere sólo, como condición $a$ priori, a los fenómenos externos" (KrV, A34/B50). De esta manera, el grado de universalidad del tiempo le conferiría a este el estatus de elemento predominante básico del conocimiento puro (Cf. Heidegger,1929, p. 40). Es, por lo demás, una consecuencia que podría desprenderse fácilmente del hecho de que "incluso nuestras ideas de las cosas espaciales son, si las tomamos en sí mismas, determinaciones o estados de nuestras mentes, y por tanto necesariamente en el tiempo" (Paton, 1936, p. 149).

Ahora bien, parece ser que la razón principal por la cual se considera prioritario al tiempo en la Crítica radica en la extensión del concepto de tiempo, ${ }^{10}$ pues este se aplicaría a un dominio de objetos mayor que el concepto de espacio. Sin embargo, esto no basta para justificar completamente que, desde el punto de vista lógico, ${ }^{11}$ el tiempo posee efectivamente dicha prioridad. En efecto, siguiendo ese modo de argumentación -i.e., si consideramos que la actividad mental es prioritariamente temporal y no espacial por el grado de universalidad-si definimos, por ejemplo, 'gato' como 'felino doméstico', habría que decir que 'gato' es principalmente 'felino' y secundariamente 'doméstico', dado que el primer concepto posee una extensión mayor que el segundo; pero 'felino' sería principalmente 'mamífero', éste último 'animal', y así sucesivamente podemos escalar el árbol de Porfirio hasta llegar al género más general: 'ente', 'ser' o 'cosa'. En consecuencia, tendríamos que decir, por transitividad, que 'gato' es prioritariamente 'cosa'. Pero todo lo que hay sería también prioritariamente 'cosa', de donde se concluye que no habría ningún objeto u orden de cosas que sea lógicamente más fundamental que otro. Así, por ejemplo, es evidente que no puede haber fenotipo sin genotipo, pero si ambos tuvieran el mismo

\footnotetext{
${ }^{10}$ Podría parecer extraño al lector que se hable del 'concepto' de tiempo en circunstancias que el mismo Kant, ya en la Estética Trascendental es enfático al señalar que el tiempo no es un concepto, sino una intuición pura, pues el concepto es universal, mientras que la intuición es singular -"tiempos diferentes son sólo partes de un mismo tiempo" (KrV, A32/B47)-. Ahora bien, lo anterior por ningún motivo implica que no podamos hablar conceptualmente sobre el tiempo; es, de hecho, el único modo que tenemos de pensarlo, analizarlo y expresarlo - del mismo modo en que hablar del concepto de libertad no implica que la libertad sea un concepto o hablar del concepto de acción no implica que la acción sea un concepto-. Esto es especialmente relevante si consideramos que nos estamos refiriendo aquí a la universalidad del tiempo y no a su carácter intuitivo singular. En este sentido, al igual que en un concepto, nos estamos refiriendo a la cantidad de objetos que caen bajo el dominio de esta forma.

${ }^{11}$ Se distingue aquí la perspectiva lógica de la perspectiva empírica, pues en estos casos se establecen órdenes de prioridad distintos. Así pues, en el orden empírico podemos decir que si definimos 'hombre' como 'animal racional', entonces el hombre es, antes que todo, animal, pues no se puede llegar a ser hombre sin haberse desarrollado un cuerpo de animal, cuyo cerebro posteriormente permitirá la racionalidad. Sin embargo, desde el orden lógico de la definición de 'hombre', no se puede sostener con la misma seguridad que este siga siendo, antes que todo, animal o racional.
} 
grado de prioridad, según el razonamiento anterior, entonces no se podría sostener dicha conexión causal. Luego, el argumento principal de la prioridad temporal no se sostiene en términos formales. En lo que sigue se mostrará que, por el contrario, existen buenas razones para sostener que en la Crítica la prioridad viene más bien de la mano del espacio.

Hacia el final de la sección dedicada al Sistema de los Principios del Entendimiento Puro Kant introduce, en la segunda edición de la Crítica, un pasaje que se titula Refutación al Idealismo. En este apartado el filósofo intenta desarrollar una prueba del mundo externo, con la finalidad de no ser identificado ni con el idealismo material de Berkeley ni con el idealismo problemático de Descartes. Lo interesante aquí es que se establece una relación entre el sentido interno y el sentido externo. El argumento, que intenta probar la existencia de los objetos externos a partir de la conciencia interna, incluye cuatro premisas: La primera, de inspiración cartesiana, es que (1) "soy consciente de mi existencia en cuanto determinada en el tiempo" (KrV, B275). En efecto, sólo a través de una introspección simple puedo percatarme de que, toda vez que pienso, existo -lo que puede ser llamado 'principio de inmanencia'- y que, dado el flujo sucesivo en el que mis pensamientos se encuentran, se infiere que mi existencia se halla igualmente determinada bajo esta condición temporal. Ahora bien continúa Kant-, (2) "toda determinación temporal supone algo permanente en la percepción" (ídem). Así pues, para que diversos contenidos representados por la conciencia puedan ser considerados como sucesivos, es necesario que haya algo permanente que opere como sujeto de los cambios, a partir de lo cual se pueda considerar una sucesión qua sucesión. De otro modo, no habría manera de distinguir un evento de otro -1lámese a esto 'principio del cambio'-. (3) "Pero ese elemento permanente -advierte Kant- no puede ser algo en mí, ya que mi propia existencia solo puede ser determinada en el tiempo mediante dicho elemento"12 (idem). Dado, entonces, que todo -incluidos mis estados mentales internos y la conciencia de mí mismo- me lo represento con una cierta duración a partir de mis representaciones, entonces se sigue que aquello permanente no puede ser algo en mí -llámese a esto 'principio de desustancialización del sujeto'-. Ahora bien -infiere Kant-, (4) "la percepción de este [elemento permanente] sólo es, pues, posible a través de una cosa exterior a mí, no a través de la simple representación de una cosa exterior a mí" (ídem), de donde Kant concluye que, "consiguientemente, la determinación temporal de mi existencia sólo es posible

\footnotetext{
${ }^{12}$ En el Prólogo de la Crítica, Kant propone modificar esta oración como sigue: "Pero este algo permanente no puede ser una intuición en mí. Pues todos los fundamentos de determinación de mi existencia que pueden hallarse en mí son representaciones y, como tales, ellas mismas necesitan un algo permanente distinto de ellas, en relación con lo cual pueda determinarse su cambio y, consiguientemente, mi existencia en el tiempo en que tales representaciones cambian" (KrV, BXXXIXn).
} 
gracias a la existencia de cosas reales que percibo fuera de mí" (KrV, B275 y s.). Más allá de si en este pasaje Kant prueba lo que intenta probar, lo cierto es que se muestra "que en realidad la experiencia externa es inmediata, que sólo a través de ella es posible, no la conciencia de nuestra propia existencia, pero sí su determinación en el tiempo, es decir, la experiencia interna" (KrV, B276 y s.). Un poco más adelante, Kant es todavía más explícito al señalar que, según lo anterior, "la experiencia interna en general sólo es posible a través de la experiencia externa" (KrV, B278 y s.). La razón principal que tiene Kant para sostener esto es que, para intuirnos a nosotros mismos, se debe ejecutar una autoafección $^{13}$ (Cf. KrV, B68 y s. y B156 y ss.), dado que sólo nos podemos conocer en cuanto fenómenos y no en cuanto a lo que somos en sí mismos. Si esto es así, entonces la conciencia de sí se ve sometida a las mismas condiciones formales que hacen posible la representación de objetos, ${ }^{14}$ i.e., a las condiciones de la experiencia externa: "la autoconciencia empírica carece de autonomía para constituirse por sí misma, y sólo puede darse, en consecuencia, sobre el trasfondo de un mundo de objetos espacio-temporales permanente" (Jáuregui, 2008, p. 99).

La conclusión de la Refutación al Idealismo se hace mucho más clara a la luz de las enseñanzas de la Deducción Trascendental en sus dos versiones. Según esta sección, la unidad objetiva de autoconciencia o apercepción trascendental hace posible la unidad subjetiva de autoconciencia -o flujo de conciencia en el sentido interno- (Cf. KrV, B139 y s.), dado que "el psiquismo no podría pensar, y menos a priori, la identidad del yo en medio de la diversidad de sus representaciones, si no tuviera presente la identidad de su acto" (KrV, A108. Énfasis propio). En este sentido, la identidad de los actos objetivantes que ejecuta la mente con sus categorías sobre el sentido externo constituye el principio unificador de la conciencia, posibilitando de este modo la conciencia empírica sucesiva de los estados mentales internos. En consecuencia, la unidad del objeto en el sentido externo ejerce en la mente una fuerza opositiva que evidencia la diferencia entre yo y el mundo, diferencia desde la cual emerge la identidad del sujeto y el objeto. De lo anterior se sigue, naturalmente, que la forma del sentido externo, es decir, el espacio, tiene una primacía respecto de la forma del sentido interno, es decir, del tiempo.

\footnotetext{
${ }^{13}$ La autoafección es la pieza clave para comprender el dinamismo epigenético del sujeto en el modelo representacional de la mente kantiano. Recuérdese que Kant no postula una 'sustancia' pensante que opere como soporte de la continuidad del Yo en el tiempo, sino más bien un conjunto de facultades y representaciones ensambladas de tal modo que constituyen el principio trascendental de autoconciencia.

14 "Las determinaciones del sentido interno tenemos que disponerlas en el tiempo precisamente del mismo modo según el cual disponemos en el espacio las de los sentidos externos" (KrV, B156).
} 


\section{ESPACIALIZACIÓN DEL TIEMPO}

Una cosa es que el espacio posea una prioridad lógica e intuitiva respecto del tiempo y otra muy distinta es que el tiempo mismo se derive trascendentalmente del espacio. En este último apartado se intentará mostrar que esta tesis encuentra un respaldo textual en la Crítica.

En la sección del Esquematismo Trascendental Kant se propone evidenciar los modos en que las categorías se temporalizan en orden a constituir el horizonte sobre el cual los objetos son representados y representables fenoménicamente ante la conciencia. El esquema es, por tanto, una determinación trascendental del tiempo (Cf. KrV, A138/B177). Dentro de los esquemas ${ }^{15}$ que Kant describe conforme a la división de las categorías, se enfocará aquí la atención en los esquemas que corresponden a la cantidad, dado que en su descripción se puede apreciar el modo según el cual concibe Kant la producción del tiempo:

"El esquema puro de la magnitud (quantitas), entendida como concepto del entendimiento, es, en cambio, el número, el cual constituye una representación que comprende la sucesiva adición de unidades homogéneas. El número no es, pues, otra cosa que la unidad de síntesis de lo diverso de una intuición homogénea en general, unidad obtenida al producir yo el tiempo mismo en la aprehensión de la intuición" (KrV, A142 y s.).

En este pasaje, Kant sostiene que el número o serie temporal ${ }^{16}$ es producido por una unidad de síntesis de la multiplicidad dada en la intuición que opera toda vez que se ejecuta el acto de aprehensión de los fenómenos. Según la Deducción Trascendental, la unidad de síntesis es la unidad categorial que está siempre presupuesta en la aprehensión. Esto último se evidencia tanto en la 'Deducción A'-específicamente en la doctrina de la triple síntesis- como en el controversial $\S 26$ de la 'Deducción B'. Ahora bien, según lo visto en la sección anterior de este trabajo, la síntesis categorial se proyecta primariamente sobre el espacio y luego es posible la determinación del sentido interno en el tiempo. En este sentido, si la

\footnotetext{
${ }^{15} \mathrm{Si}$ bien Kant no es explícito sobre el número de los esquemas, y dado que es incluso posible pensar que el autor presenta un esquema para la cantidad, uno para la cualidad y tres para la relación y la modalidad, parece ser que la distinción es simétrica con la tabla de las categorías. En efecto, cuando Kant se refiere al tiempo en cuanto número, sostiene que no es otra cosa que "la unidad de síntesis de lo diverso" (KrV, A142 y s./B182). Esta expresión podría hacer alusión a las categorías de unidad, pluralidad y totalidad, en tanto que esta última surge a partir de la unificación de la diversidad. Respecto de los esquemas de cualidad, Kant es explícito al señalar la realidad, la negación y la oposición (Cf. $\mathrm{KrV}, \mathrm{A} 143 / \mathrm{B} 182)$, lo cual parece ser una referencia a las categorías de realidad, negación y limitación. Según todo lo anterior, entonces, sería posible hablar de doce esquemas.

${ }^{16}$ Se considera aquí 'número' y 'serie' temporal como términos sinónimos, dado que ambos hacen referencia al quantum del tiempo, si bien el pasaje es oscuro y no está planteada esta idea explícitamente.
} 
aprehensión es condición necesaria del número del tiempo y, a su vez, las categorías son condiciones necesarias para la aprehensión, y esta unidad categorial se dirige antes que todo a la forma del sentido externo, i.e., al espacio, entonces se sigue que la producción originaria del tiempo descansa constitutivamente sobre el espacio.

Hasta aquí sólo se ha explicado que, según la Refutación al Idealismo, el espacio tiene una primacía por sobre el tiempo, y que el Esquematismo sugiere, por su parte, que la misma producción de la serie temporal deriva de la acción del entendimiento sobre el espacio. Sin embargo, queda todavía una cuestión por resolver: si la Estética Trascendental señala que una de las propiedades características del espacio es la simultaneidad, mientras que la del tiempo es la sucesión (Cf. KrV, A31/B47), entonces, ¿cómo puede emerger lo sucesivo desde lo simultáneo? Una lectura a las Analogías de la Experiencia podría arrojar luz sobre esta interrogante.

En las Analogías Kant describe con detalle los principios que se siguen producto de la esquematización de las categorías de relación, i.e., sustancia, causalidad y comunidad. Estos principios se refieren, no a la forma de la intuición, sino a la existencia de los objetos en el tiempo, de modo tal que regulan sus relaciones, a diferencia de los Axiomas de la Intuición y las Anticipaciones de la Percepción que, más bien, constituyen sus intuiciones. Lo interesante aquí es que las tres analogías se comunican entre sí para dar origen a un único principio, a saber, que "la experiencia sólo es posible mediante la representación de una necesaria conexión de las percepciones" (KrV, B218). En efecto, tal y como se señala en la premisa (2) de la Refutación al Idealismo, para ser conscientes de la sucesión, es necesario algo que permanezca en los cambios, y en la primera analogía -principio de la permanenciaKant señala precisamente que esto permanente es el tiempo mismo considerado como sustrato (Cf. KrV, A182/B225). Pero el principio de permanencia se define también en función de las dos siguientes analogías, i.e., a partir del principio de la sucesión temporal y de la simultaneidad. La relación con la primera es que, así como sólo se puede percibir la sucesión a partir de algo permanente en la intuición, lo permanente, a su vez, sólo puede distinguirse en la conciencia empírica como tal si cambia. Como dice Kant, paradójicamente, "solo lo permanente (la sustancia) cambia; lo mudable no sufre cambio alguno, sino modificación, ${ }^{17}$ ya que algunas determinaciones desaparecen y otras aparecen" (KrV, A187/B231). Pero también hay una relación entre los principios anteriores y la simultaneidad. En efecto, por un lado, para tener conciencia de dos o más datos como simultáneos -por ejemplo, de la pantalla y del teclado de mi computador, además del escritorio sobre el cual

\footnotetext{
${ }^{17}$ Ribas traduce Veränderung por 'cambio' y Weschel por 'modificación'. Caimi, por el contrario, traduce estas expresiones correspondientemente por 'alteración' y 'cambio'. Ninguna de las traducciones parece ser exacta, pero la idea central a considerar aquí es que aquello que permanece en el tiempo sufre sólo cambios accidentales, mientras que aquello que no permanece es simplemente reemplazado por otras características.
} 
este se encuentra, etc.- es necesario que ambos eventos permanezcan dentro de una misma unidad temporal en la cual los percibo. Por otro lado, si causa y efecto no son concebidos en mutua interacción -principio de la simultaneidad o comunidadconforme a la cual a toda acción le corresponde una determinada reacción, entonces sería imposible representarse algo como causa y algo como efecto, dado que serían intuiciones desconectadas entre sí; sería, por lo tanto, imposible representarse el cambio y, en consecuencia, sería indiscernible lo permanente en las apariencias y, de este modo, sería igualmente imposible la conexión de las percepciones -i.e., la experiencia-. Por lo tanto, las tres reglas a priori de las Analogías de la Experiencia deben ser entendidas como tres momentos de un mismo principio, momentos que pueden ser distinguidos, mas no comprendidos los unos sin los otros.

Como consecuencia de lo anterior, podemos apreciar que la sucesión temporal sólo es posible desde la comunidad de sustancias, es decir, desde la aprehensión de la coexistencia de objetos permanentes en el espacio. Por lo tanto, se explica sin mayores problemas cómo puede emerger, en la Crítica, lo sucesivo desde lo simultáneo. Dentro del modelo representacional epigenético de la razón pura se presenta, entonces, la exigencia teórica de la espacialización del tiempo.

\section{CONCLUSIÓN}

En este trabajo se ha mostrado que (1) el enfoque epigenético de la Crítica permite descubrir con mayor exactitud y alcance los mecanismos que operan a nivel trascendental. Esto se logra a partir de una comprensión de que las estructuras puras a priori surgen como producto de una actividad mental que tiene lugar con ocasión de la receptividad de datos sensibles, análogamente al modo en que en los seres vivos emergen los rasgos propios en la medida en que su genoma entra en contacto con el ambiente. Dado que la epigénesis requiere siempre de un orden jerárquico, se estimó necesario investigar si acaso existe una primacía del espacio respecto del tiempo. Así pues, se mostró que (2) el principal argumento que se ha presentado en favor de la primacía del tiempo no se sostiene y que, en cambio, hay buenas razones -que emanan de distintos pasajes de la Crítica-para pensar que es el espacio el que más bien prima por sobre el tiempo. Finalmente, (3) se intentó evidenciar que no sólo existe una prioridad del espacio, sino que además el tiempo mismo es producido originariamente desde las relaciones espaciales de simultaneidad.

Como se puede apreciar, los argumentos aquí presentados descansan completamente en una lectura conceptualista ${ }^{18}$ de la Crítica de la razón pura, es decir, en una interpretación según la cual toda percepción, todo estado empírico subjetivo o

\footnotetext{
${ }^{18}$ En este sentido, seguimos la noción sellarsiana del Mito de lo Dado que es integrada en una lectura conceptualista de la Crítica de la razón pura por McDowell (1994) y (2006).
} 
toda determinación del sentido interno, para ser conectado a una conciencia, requiere como trasfondo de una síntesis categorial proyectada sobre el sentido externo. Sin embargo, tal y como lo han hecho ver diversos autores, ${ }^{19}$ en la Crítica hay un número importante de pasajes en los que parece sugerirse que existe una unidad intuitiva previa a la síntesis categorial. ${ }^{20} \mathrm{Si}$ esto es así, la argumentación presentada en este trabajo queda invalidada. No obstante, se considera aquí que presentar una lectura no-conceptualista de la Crítica nos llevaría a problemas sistemáticos agudos que pondrían en jaque algunas de las afirmaciones más identitarias del pensamiento de Kant. Naturalmente, esto último excede ampliamente el espacio del que se dispone en esta investigación.

Hay otro tema que quedaría pendiente para una futura investigación: si la interpretación sobre la espacialización del tiempo en la Crítica es correcta, entonces habría que preguntarse cuál es el rol que cumple la facultad de juzgar reflexionante en la constitución de las intuiciones formales de espacio y tiempo. Más precisamente, se trataría de mostrar la función que en ello cumplen los conceptos de reflexión introducidos por Kant en el apéndice titulado Anfibología de los Conceptos de Reflexión, dentro de los cuales se cuentan: identidad-diferencia, concordancia-oposición, interior-exterior y determinable-determinación.

\section{BIBLIOGRAFÍA}

Allais, L. (2009): "Kant, Nonconceptual Content and the Representation of Space", Journal of the History of Philosophy, Vol. 47, 3, pp. 383-413.

Allison, H. (1973): The Kant-Eberhard Controversy: The John Hopkins University Press.

De Pierris, G. (1987): “Kant and Innatism”, Pacific Philosophical Quarterly, Vol. 68, pp. 285-305.

Franck, J. F. (2007): "El problema del innatismo en Antonio Rosmini (parte II)", Sapientia Vol. LXII, pp. 54-76.

Kant, I. Crítica de la razón pura, Edición bilingüe alemán-español. Traducción, estudio preliminar y notas de Mario Caimi, Ed. Fondo de Cultura Económica, México, D.F., 2009.

Kant, I. Crítica de la razón pura. Traducción, introducción, notas e índices de Pedro Ribas, Editorial Taurus, México, D.F., 2006.

\footnotetext{
${ }^{19}$ Por ejemplo, Hanna (2005), Allais:(2009), Land (2015), entre muchos otros.

${ }^{20}$ Por ejemplo, KrV, A89 y s./B122 y s., B132, B145, B161n, etc. Para una revisión de estos y otros pasajes problemáticos, véase Lazos (2014).
} 
Kant, I. Critique of the Power Jugdement. Traducción de Paul Guyer y Eric Matthews. Cambridge University Press, Cambridge, 2002.

Kant, I. Lectures on metaphysics, Traducción de Karl Ameriks y Steve Naragon. Cambridge University Press, Cambridge, 1997.

Kant, I. Notes and fragments [Reflexionen], Traducción de Curtis Bowman, Paul Guyer y Frederick Rauscher, Cambridge University Press, New York, 2005.

Kant, I. On a discovery whereby any new critique of pure reason is to be made superfluous by an older one [Respuesta a Eberhard], en Theoretical Philosophy 1755-1770, Traducción y edición de David Walford, Cambridge University Press, Cambridge, 1992.

Kant, I. On the form and principles of the sensible and the intelligible world, en Theoretical [Dissertatio] Philosophy 1755-1770, Traducción y edición a cargo de David Walford, Cambridge, 1992: Cambridge University Press,.

Hanna, R. (2005) “Kant and Nonconceptual Content”, European Journal of Philosophy, 13, 2, pp. 247-290.

Heidegger, M. (1929) Kant y el problema de la metafísica, Traducción de Gred Ibscher Roth, México D.F. 2013: FCE.

Jáuregui, C. (2008) Sentido interno y subjetividad: Un análisis del problema del autoconocimiento en la filosofía trascendental de I. Kant, Buenos Aires: Prometeo Libros.

Kemp Smith, N. (1923): A Commentary to Kant's 'Critique of pure reason', Second edition, revised and enlarged, Humanities Press, USA, 1962.

Land, T. (2015): "Nonconceptualist Readings of Kant and the Transcendental Deduction", Kantian Review, Vol. 20, Issue 1, pp. 25-51.

Lazos, E. (2014): Disonancias de la Crítica: Variaciones sobre cuatro temas kantianos, México, D.F. Universidad Autónoma de México.

Lorenz, K. (1941): "La teoría kantiana de lo apriorístico desde el punto de vista de la biología actual” en LORENZ, K. y WUKETITS, F. M. (1984) La evolución del pensamiento, pp. 89-116.

Mcdowell, J. (1994): Mind and World: Harvard University Press.

Mcdowell, J (2006): "Sensory Consciousness in Kant and Sellars", Philosophical Topics, Vol. 34, 1-2, pp. 311-326.

Olson, M. (2017): “The Metaphysics of the Epigenesis of Reason: On Jennifer Mensch's Kant's Organicism”, Philosophy Today, Vol. 61, Issue 3, pp. 793-799.

Paton, H. J. (1936): Kant's Metaphysic of experience, vols. I y II. Londodn: Unwin Brothers.

Vecchi, D. Y Hernández, I. (2015), "Epigénesis y preformacionismo: radiografía de una antinomia inconclusa”, Scientiae Studia, Vol. 13, 3, pp. 577-597.

Waxman, W. (1991): Kant's Model of the Mind: A New Interpretation of Transcendental Idealism, New York: Oxford University Press.

Zoeller, G. (1989): "From Innate to “A Priori”: Kant's Radical Transformation of a CartesianLeibnizian Legacy”, The Monist, Vol. 72, 2, pp. 222-235. 